\title{
Rheology of F-actin solutions determined from thermally driven tracer motion ${ }^{\mathrm{a})}$
}

\author{
T. G. Mason ${ }^{b)}$ \\ Corporate Strategic Research, ExxonMobil Research and Engineering Co., \\ Route 22 East, Annandale, New Jersey 08801 \\ T. Gisler \\ Universität Konstanz, Fakultät für Physik, Postfach 5560, \\ D-78457 Konstanz, Germany \\ K. Kroy \\ École Sup. de Physique et Chimie Industrielles, 10 rue Vauqueline, \\ F-75231 Paris Cedex 05, France \\ E. Frey and D. A. Weitz \\ Department of Physics and DEAS, Harvard University, 29 Oxford Street, \\ Cambridge, Massachusetts 02138
}

(Received 7 January 2000; final revision received 17 April 2000)

\begin{abstract}
Synopsis
We report measurements of the frequency-dependent complex shear modulus of semidilute F-actin solutions based on optical observations of the thermally excited motion of monodisperse tracer microspheres. Because the tracer spheres cause incident laser light to be strongly scattered, we determine their average motion using diffusing wave spectroscopy. From the measured mean square displacement, we extract the retardation spectrum of the actin solution using a regularized fit based on a discretized model involving a linear superposition of harmonically bound Brownian particles. At an actin concentration of $C=1.2 \mathrm{mg} / \mathrm{ml}$ and for microspheres of radius $a=0.8 \mu \mathrm{m}$, we find that the complex modulus exhibits a dominant low frequency plateau modulus and a high frequency rise with the loss modulus dominating above a crossover frequency. Over a limited range of frequencies well above the crossover frequency, the magnitude of the high frequency storage modulus $G^{\prime}(\omega)$ is consistent with the power law scaling $\omega^{3 / 4}$. The observed gradual crossover appears to be at odds with previous theoretical predictions, but it corresponds to a simple structure of the retardation spectrum.
\end{abstract}

\section{INTRODUCTION}

Understanding the origin of the elasticity of the cytoplasm of living cells is an important challenge. Because a tremendous diversity of cell types exists, the possibility of identifying a single universal mechanism which gives rise to the cytoplasmic elasticity is

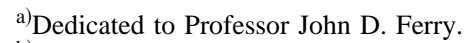

b) Author to whom correspondence should be addressed; electronic mail: tgmason@erenj.com

Konstanzer Online-Publikations-System (KOPS)

URL: http://www.ub.uni-konstanz.de/kops/volltexte/2008/5390/

URN: http://nbn-resolving.de/urn:nbn:de:bsz:352-opus-53903 
remote. However, the abundance of the monomeric globular protein G-actin, which aggregates, or polymerizes, at physiological salt concentrations to form F-actin, long rodlike filaments having a typical length $L \approx 10 \mu \mathrm{m}$ and diameter of $8 \mathrm{~nm}$, has led to the supposition that F-actin may be the dominant contributor to the cytoplasmic elasticity in many cell types. One way to explore the validity of this supposition is to study the rheology of purified F-actin solutions in vitro at physiological concentrations and compare this to the rheology of the cytoplasm within living cells. Such rheological measurements of F-actin solutions should also provide insight into the general frequency dependence of the linear viscoelastic moduli of rigid semiflexible polymers having long persistence lengths $L_{p}$.

The exploitation of a separation protocol for obtaining highly purified actin [Spudich and Watt (1971)] has led to numerous in vitro measurements of F-actin shear viscoelasticity, including mechanical rheometry [Janmey et al. (1994); Xu (1997)] and real-space microrheometry [Ziemann et al. (1994); Amblard et al. (1996); Gittes et al. (1997)]. However, large differences in the reported magnitudes and frequency dependencies of the viscoelasticities have appeared. For instance, at an actin concentration of $C=48 \mu \mathrm{M}$ $=2 \mathrm{mg} / \mathrm{ml}$, a low-frequency plateau elastic storage modulus $G_{p}^{\prime}$ measured mechanically, has been reported to be in the range of $G_{p}^{\prime} \approx 10^{3}-10^{4}$ dynes $/ \mathrm{cm}^{2}$ [Janmey et al. (1994)], whereas a different group has reported a much lower $G_{p}^{\prime} \approx 20$ dynes $/ \mathrm{cm}^{2}$, at the same $C[\mathrm{Xu}(1997)]$. The very large values of $G_{p}^{\prime}$ have been shown to arise from artifacts in the preparation of actin that lead to crosslinking, and there is now general agreement that the smaller value of $G_{p}^{\prime}$ does describe uncrosslinked F-actin solutions [Xu et al. (1998a, 1998b)]. Differences also appear in the high frequency behavior of the storage modulus $G^{\prime}(\omega)$ and loss modulus $G^{\prime \prime}(\omega)$, which have been reported to both vary as $\omega^{1 / 2}$ between $1 \mathrm{~s}^{-1} \leqslant \omega \leqslant 10 \mathrm{~s}^{-1}$ [Ziemann et al. (1994)] or alternatively as $\omega^{3 / 4}$ between $10^{1} \mathrm{~s}^{-1} \leqslant \omega \leqslant 10^{2} \mathrm{~s}^{-1}$ [Gittes et al. (1997)]. Such frequency dependencies imply that the creep compliance $J(t)$, an equivalent representation for the viscoelasticity in the time domain, should vary as $J(t) \sim t^{1 / 2}$ or alternatively as $t^{3 / 4}$ at early times. To help resolve these differences, we carefully consider the measured high-frequency behavior of the storage and loss moduli and compare these with the predicted scaling.

In a recent publication [Gisler and Weitz (1999)], we have reported a universal scaling form for the frequency-dependent linear viscoelastic moduli of F-actin solutions based on many diffusing wave spectroscopy (DWS) measurements of microsphere tracer motion. The essential proposed form includes a low frequency plateau in the storage modulus that may arise due to filament entanglements and some degree of filament crosslinking and a high frequency rise in both the storage and loss moduli of $\omega^{3 / 4}$ due to the intrinsic bending rigidity of the filaments. Their scaling form also includes a $\omega^{1 / 3}$ dependence to account for the observed gradual crossover between these low and high frequency limits. By contrast, in this paper, rather than focus on the universality of the shape of the viscoelastic spectra, we consider several representative measurements of the tracer motion in detail and extract the retardation spectrum of the F-actin solution using a regularized fit to a linear superposition of harmonically bound Brownian particles having logarithmically spaced, discretized time constants. From the regularized fits, we verify the previously reported behavior of the storage and loss moduli as a function of frequency without assuming any power law scaling form a priori in the analysis.

\section{EXPERIMENT}

In order to extract the linear rheological properties of the F-actin solution from the motion of the tracer microspheres, one must ensure strong scattering by the microspheres 
without greatly disturbing the F-actin network. Rabbit G-actin was purified and then stored in a buffer consisting of $2 \mathrm{mM}$ Tris-Cl, $0.2 \mathrm{mM}$ ATP, $0.5 \mathrm{mM}$ dithiothreitol, 0.1 $\mathrm{nM} \mathrm{CaCl} 2$ at a temperature of $T=-80^{\circ} \mathrm{C}$. Immediately before the DWS measurements, the frozen G-actin solution was thawed to $T=37^{\circ} \mathrm{C}$ and the concentration $C$ $=1.2 \mathrm{mg} / \mathrm{ml}$ of the actin was set by dilution with the buffer and measured using ultraviolet-visible spectroscopy. This concentration lies nearly an order of magnitude above the overlap concentration associated with the swept-out volume of the rotationally diffusing actin filaments. Surface-modified polystyrene latex particles of radius $a$ $=0.8 \mu \mathrm{m}$ were added to a volume fraction of $\phi=0.01$. This volume fraction was chosen to be small enough that the spheres would not greatly affect the polymer solution rheology, yet large enough to ensure strong light scattering, so that the scattering from the spheres completely dominates that from the actin. The G-actin was polymerized by adding $75 \mathrm{mM} \mathrm{MgCl}_{2}{ }^{1}$ Phalloidin was added to a concentration of $1.2 \mathrm{C}$ to stabilize the actin filaments against depolymerization. The resultant filaments were about $L$ $\approx 10 \mu \mathrm{m}$ long, as determined from fluorescence microscopy. The size of the spheres was chosen to be large enough that $2 a \gg \xi$, the characteristic mesh size, yet the spheres were chosen to be small enough that thermal energy would drive sufficient sphere motion that a substantial decay in the correlation function could be measured. This was facilitated by the use of diffusing-wave spectroscopy, which is sensitive to motion over very small length scales. Following the addition of the salt, the samples were loaded into glass cells a few millimeters thick and incubated at $T=4{ }^{\circ} \mathrm{C}$ for $6 \mathrm{~h}$. No aggregation of the microspheres was observed microscopically following the salt and temperature changes. The concentration of F-actin is below that associated with observations of birefringence [Xu (1997)], so the F-actin structure is disordered.

Diffusing wave spectroscopy [Weitz and Pine (1992)] was performed in the transmission geometry using an etalon-equipped argon ion laser at a wavelength $\lambda$ $=514.5 \mathrm{~nm}$. Mean-square displacements, $\left\langle\Delta r^{2}(t)\right\rangle$, of the tracer spheres were calculated from the measured correlation function $g_{2}(t)$ using a transport mean free path $l^{*}$ determined by a separate measurement of the transmitted light intensity relative to that of a sample of known $l^{*}$. All measurements were performed at room temperature.

\section{RESULTS}

The measured mean square displacements as a function of time for the tracer spheres in the F-actin solution for two independent trials at $C=1.2 \mathrm{mg} / \mathrm{ml}$ and $a=0.8 \mu \mathrm{m}$ are coplotted in Fig. 1. The initial rise at early times is not purely diffusive, but is slightly subdiffusive, with a slope on the log-log plot of less than unity. Toward longer times, the mean square displacement becomes increasingly subdiffusive, reflecting the inhibited diffusion of the spheres by the surrounding actin filaments, until finally the mean square displacement saturates to a plateau, indicating that the spheres are spatially confined by the actin filaments. The spheres are limited on average to displacements of only tens of nanometers, much less than a sphere diameter, under room-temperature thermal excitations. Out of many possible measured mean square displacements, some of which exhibit significant variations from one trial to the next, we have selected these for analysis because they have minimal noise at very early and long times and they show good reproducibility, differing slightly only at the longest and shortest times.

\footnotetext{
${ }^{1}$ The print version incorrectly reads $75 \mathrm{nM} \mathrm{MgCl} 2$.
} 


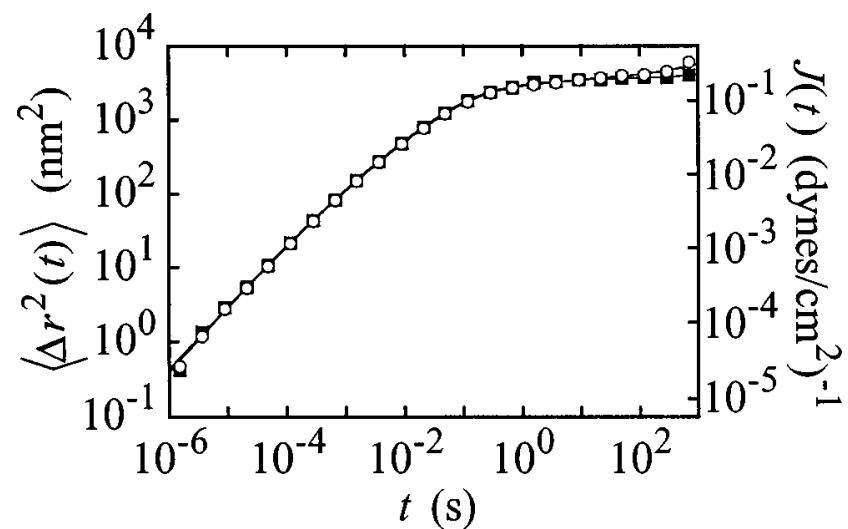

FIG. 1. Mean square displacement as a function of time $\left\langle\Delta r^{2}(t)\right\rangle$ for polystyrene microspheres of radius $a$ $=0.8 \mu \mathrm{m}$ at a volume fraction of $\phi=0.01 \mathrm{in}$ an F-actin solution at a concentration of $C=1.2 \mathrm{mg} / \mathrm{ml}$. The open circles and solid squares represent two different trials of diffusing wave spectroscopy measurements, and only every tenth data point is shown for clarity. The corresponding creep compliance $J(t)$, calculated from the mean square displacement using Eq. (2), is shown on the right axis. The solid lines represent the results of a regularized linear fit using Eq. (3).

\section{ANALYSIS}

The generalized Stokes-Einstein relation connects the time-dependent mean square displacement of the probe spheres to the linear viscoelasticity of the complex fluid in which they are suspended. Assuming that the local viscoelasticity surrounding the spheres is the same as the macroscopic viscoelasticity, the viscoelastic spectrum $\tilde{G}(s)$ as a function of Laplace frequency $s$ is [Mason and Weitz (1995); Mason et al. (1997)]

$$
\tilde{G}(s)=\frac{k_{B} T}{\pi a s\left\langle\Delta \tilde{r}^{2}(s)\right\rangle},
$$

where $k_{B}$ is Boltzmann's constant and the tilde denotes the unilateral Laplace transform. This equation results from a generalized Langevin equation which describes the spheres' motion in an isotropic incompressible viscoelastic continuum, consistent with energy equipartition and the fluctuation-dissipation theorem, where the sphere inertia has been neglected. Since the flow field of a fluid having an unknown viscoelasticity around a sphere is not known, we have assumed the Stokes relation for a viscous fluid with no-slip boundary conditions can be generalized to all $s$.

Given Eq. (1) and the simple relationship between the shear modulus and the shear creep compliance in the Laplace frequency domain, $\tilde{J}=1 /(s \tilde{G})$ [Bird et al. (1977); Ferry (1980)], it is straightforward to show that the time-domain creep compliance $J(t)$ is directly proportional to $\left\langle\Delta r^{2}(t)\right\rangle$

$$
J(t)=\frac{\pi a}{k_{B} T}\left\langle\Delta r^{2}(t)\right\rangle .
$$

This creep compliance essentially represents the ratio of the temporally evolving strain, or displacement of the sphere in the solution, to an equilibrium Brownian stress and is completely equivalent to the frequency-domain representation of the modulus. This is the Brownian analogy of a mechanical experiment in which a constant shear stress is imposed on the material and $J(t)$ is obtained by dividing the resulting measured strain by 
this stress, provided both remain in the linear regime. The primary advantage of choosing $J(t)$ as the linear viscoelastic representation is that it is directly proportional to the measured $\left\langle\Delta r^{2}(t)\right\rangle$; no transformation to the frequency domain or fitting of the data are needed to properly report the material's linear viscoelastic response. Thus, it is trivial to compute the creep compliance of the F-actin solution; the results shown in Fig. 1 (right axis labels).

There are many known ways to obtain the linear frequency-dependent storage and loss moduli from the creep compliance, including various estimation methods [Ferry (1980)]. However, the very large temporal dynamic range and logarithmic spacing of the measured mean square displacement can make some of these methods, especially the direct transform methods, difficult to implement. Moreover, noise in the mean square displacement and rapid changes in the logarithmic slope of the mean square displacement can reduce the accuracy of estimation methods. In order to avoid these potential problems, we choose to calculate the storage and loss moduli from the retardation spectrum $L(\tau)$ determined by a regularized fit of the creep compliance using a set of impartial basis functions. The functional form for the fits is a linear superposition of basis functions that exhibit short time diffusion and a long time plateau

$$
J(t)=\sum_{n=1}^{N} L_{n}\left(1-e^{-t / \tau_{n}}\right),
$$

where $N$ is the number of terms (which is kept much smaller than the number of measured data points), $\tau_{n}$ are fixed to be logarithmically spaced, and the coefficients $L_{n}$ of the basis functions which yield the retardation spectrum are allowed to vary subject to a smoothness constraint [Press et al. (1992)]. The smoothness constraint is required to avoid rapid unphysical variations in the fitting parameters due to the very broad minimum in the multidimensional $\chi^{2}$ landscape. In rheological terminology, this analysis is simply a Voigt decomposition of the creep compliance [Ferry (1980)], but there is an interesting physical analogy with the optical measurement: the same basis functions describe the mean square displacements of harmonically bound Brownian particles.

The regularized fits to the creep compliance using Eq. (3) with $N=25$ (much less than the 250 measured data points) are shown as the lines in Fig. 1 and are essentially indistinguishable from the data. These fits essentially determine the relaxation spectrum of the F-actin solution, as plotted in Fig. 2. There is good agreement between the values of $L(\tau)$ obtained from the two independent fits from $10^{-6} \mathrm{~s} \leqslant \tau \leqslant 10^{1} \mathrm{~s}$; but there are significant deviations in the values at the longest times due to the slight, but noticeable difference in the upturns of the creep compliance. The rise at early times follows $L(\tau)$ $\sim \tau^{0.85}$ (shown by the solid line) over the range $10^{-6} \mathrm{~s} \leqslant \tau \leqslant 10^{-2} \mathrm{~s}$. This is consistent with the calculation of the smoothed logarithmic slope of the mean square displacements, $\alpha(t)=d \ln \left\langle\Delta r^{2}(t)\right\rangle / d \ln t$, shown in Fig. 3. The logarithmic slope at the shortest time is about $\alpha=0.82$ at $t=3 \times 10^{-5} \mathrm{~s}$, the shortest time at which the noise in the mean square displacement permits a calculation of the logarithmic slope with adequate certainty. The peak in $L(\tau)$ at $\tau_{0}=1 / \omega_{0} \approx 10^{-1} \mathrm{~s}$, indicates the primary mode of elastic retardation of the high frequency viscous relaxation. The subsequent decrease in $L(\tau)$ at longer $\tau$ indicates that the primary crossover between viscous and elastic behavior has occurred at earlier times and the minimum in $L(\tau)$ corresponds to the flattest part of the long time plateau in the creep compliance. The final upturn in $L(\tau)$ is indicative of an ultimate long time relaxation that lies beyond the times we have probed. 


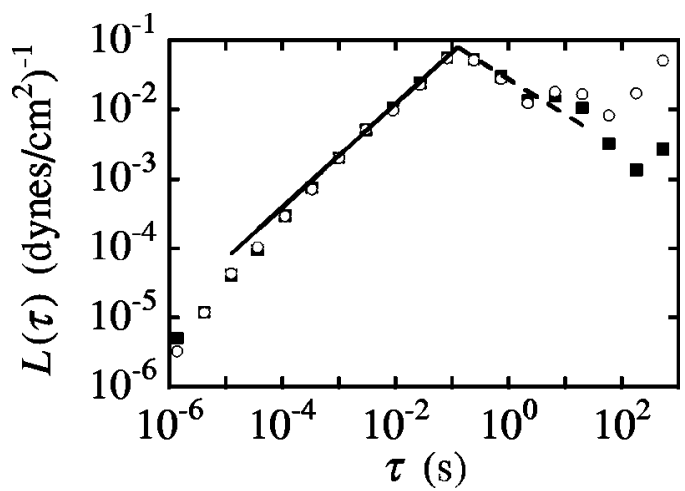

FIG. 2. Retardation spectra $L(\tau)$ of an F-actin solution at a concentration $C=1.2 \mathrm{mg} / \mathrm{ml}$ determined from the regularized fits to the mean square displacements shown in Fig. 1 (open circles and solid squares). The retardation spectra for the two trials are similar up to $\tau \approx 10 \mathrm{~s}$, after which they differ significantly due to the differences in the mean square displacements at the longest times. Below the kink at $\tau^{*} \approx 10^{-1} \mathrm{~s}$, the regularized fit scales as $\tau^{3 / 4}$ (solid line), and immediately above the kink it can be described by $\tau^{-1 / 2}$ (dashed line).

Once $L_{n}$ have been determined from the regularized fit, the storage and loss moduli are calculated by taking the real and imaginary parts of the complex modulus

$$
G^{*}(\omega)=\left(\sum_{n=1}^{N} \frac{L_{n}}{1+i \omega \tau_{n}}\right)^{-1} .
$$

Because this relationship is exact, the uncertainty in the transformation from the time to the frequency domain through regularized fitting lies only in the well-known compromise between the goodness of the fit and the degree of smoothness of the $L_{n}$. We allow at most a $1 \%$ increase in $\chi^{2}$ when we enforce the smoothness constraint. Finally, Eqs. (3) and (4) ensure that $G^{\prime}(\omega)$ and $G^{\prime \prime}(\omega)$ obey the Kramers-Kronig relations.

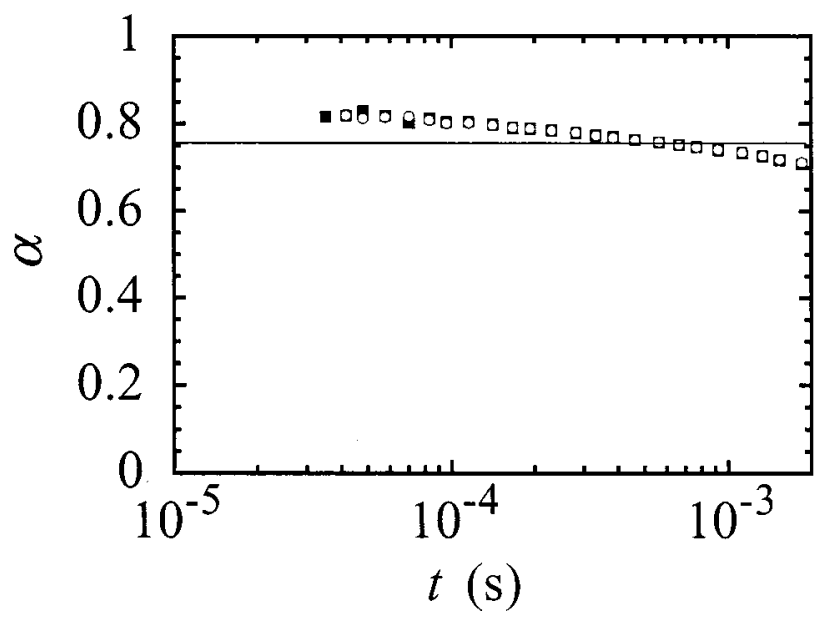

FIG. 3. The local logarithmic slope $\alpha$ of the mean square displacement as a function of time $t$ calculated from the mean square displacements shown in Fig. 1 by smoothing over the adjacent three points on either side. The local logarithmic slope rises above $3 / 4$ at the earliest times. 


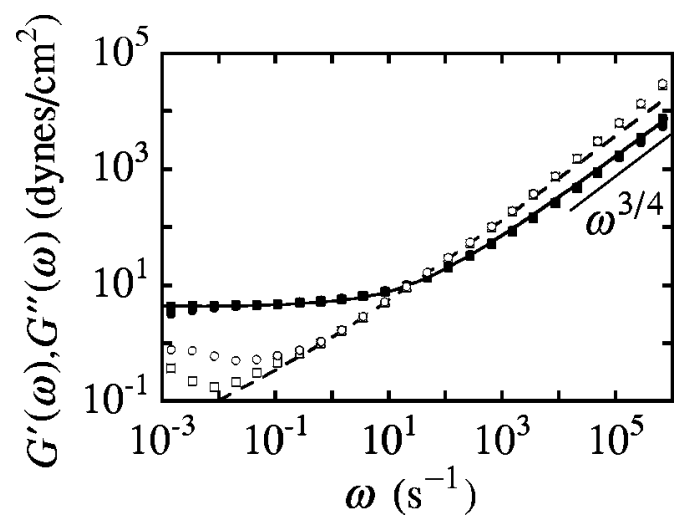

FIG. 4. The storage moduli (solid symbols) and loss moduli (open symbols) of an F-actin solution at a concentration $C=1.2 \mathrm{mg} / \mathrm{ml}$ for two different trials (circles and squares) calculated using Eq. (4) and the retardation spectra shown in Fig. 2. At high frequencies, the loss modulus dominates and the storage modulus exhibits $G^{\prime}(\omega) \sim \omega^{3 / 4}$ for $\omega \geqslant 10^{4} \mathrm{~s}^{-1}$. By contrast, at low frequencies, the storage modulus dominates with a plateau behavior, and the loss modulus goes through a minimum. Also shown are the storage modulus (solid line) and the loss modulus (dashed line) calculated from an idealized retardation spectrum $L(\tau)$ that obeys $L\left(\tau<\tau^{*}\right) \sim \tau^{3 / 4}$ and $L\left(\tau>\tau^{*}\right) \sim \tau^{-1 / 2}$ shown by the lines in Fig. 2. These moduli agree well with the measured moduli in the gradual crossover region.

The calculated $G^{\prime}(\omega)$ and $G^{\prime \prime}(\omega)$ based on the retardation spectra are shown for the two trials in Fig. 4. Here, the general features of the frequency-dependenct viscoelastic moduli of disordered semiflexible filaments well above the overlap concentration become evident. The dominant high frequency behavior is lossy. There, the ratio of the loss modulus of the solution to the loss modulus of pure water is only about a factor of 4 at $\omega=10^{6} \mathrm{~s}^{-1}$. By contrast, the high frequency storage modulus is consistent with $G^{\prime}(\omega) \sim \omega^{3 / 4}$ only for $\omega \geqslant 10^{4} \mathrm{~s}^{-1}$. The locally calculated logarithmic slope of $G^{\prime}(\omega)$ is clearly less than $3 / 4$ at lower frequencies. The dominant low frequency behavior is an elastic plateau with a plateau value of $G_{p}^{\prime}=5$ dynes $/ \mathrm{cm}^{2}$; this value is so low that the F-actin solution yields and flows under gravitational force if the sample cell is tipped sideways. Overall, there is good agreement between the two calculations: the only significant deviation is in the low frequency loss modulus, and this deviation is due to differences between the measured final upturns in the creep compliance at the very longest times. These differences in the final low frequency relaxation may be due to the ultimate breakup of the "entangled" filament cages in which the microspheres are trapped, and this glassy behavior could be strongly shear-history dependent.

\section{DISCUSSION}

Recently, there has been substantial progress in our theoretical understanding of the viscoelastic properties of (strongly entangled) semiflexible polymer solutions [for a review see, e.g., Frey et al. (1999)]. There seems to be a consensus regarding the physical principles which govern the elastic behavior at intermediate frequencies and the viscoelastic behavior at very high frequencies. In the following, we discuss our experiments in relation to theory and point out aspects of the data that raise some interesting theoretical questions. 


\section{A. Plateau modulus}

Two widely different single-polymer models of the mean-field type have been proposed to explain the plateau modulus. In the affine model one assumes that the contour of each filament in solution follows on some coarse-grained scale affinely the macroscopic deformation [MacKintosh et al. (1995)]. The resulting plateau modulus scales like $G_{p}^{\prime}$ $\sim C^{11 / 5}$ and leads to an absolute value of the order of $10 \mathrm{~Pa}$ for a solution of about 1 $\mathrm{mg} / \mathrm{ml} \mathrm{F-actin;} \mathrm{such} \mathrm{high} \mathrm{values} \mathrm{are} \mathrm{at} \mathrm{odds} \mathrm{with} \mathrm{recent} \mathrm{experiments} \mathrm{of} \mathrm{F-actin} \mathrm{solutions}$ [Hinner et al. (1998)]. The second approach to explain the observed plateau modulus employs the tube picture in which the effect of the neighboring polymers on a test filament is represented by a confinement to a cylindrical tube of diameter $d$. Combining scaling laws for the confinement energy of a semiflexible polymer [Helfrich and Harbich (1985); Odijk (1986)], which yield a scaling for the entanglement length $L_{e}$ $\sim\left(d^{2} L_{p}\right)^{1 / 3}$, and the concentration dependence of the tube diameter $d$ [Semenov (1986)], $d \sim \xi^{2} / L_{e}$, it was predicted [Isambert and Maggs (1996)] that the plateau modulus should scale as $G_{p}^{\prime} \sim C^{7 / 5}$. The basic idea can also be reformulated in terms of a virial expansion using tubes as the basic constituents of the polymer solution [Hinner et al. (1998)]. The tubes are coarse grained representations of the polymers, with the short wavelength fluctuations averaged out. One assumes that the shear modulus-in close analogy to the osmotic pressure - can be written in terms of a virial expansion in the polymer concentration: $G_{p}^{\prime}=(3 / 5) k_{B} T C(1+2 B C+\ldots)$. According to Onsager [Onsager (1949)], the second virial coefficient can be estimated as $B=\pi d L^{2} / 4$, corresponding to a number $B C=L /\left(2 L_{e}\right)$ of mutual collisions of the tubes. However, to stay consistent with the assumption that short wavelength modes have already relaxed, we subtract half the collision length at each end from $L$ to account for the reduced efficiency of dangling ends in the entanglement process. The above relation between the second virial coefficient and the collision length thus becomes $2 B C=L / L_{e}-1$ $=\pi C d\left(L-L_{e}\right)^{2} / 2$. For entangled solutions one thus finds $G_{p}^{\prime}=9 k_{B} T /\left(5 \xi^{2} L_{e}\right)$, where far from the entanglement transition $L_{e}$ and $d$ take their asymptotic values $L_{e}$ $=0.58 \xi^{4 / 5} L_{p}^{1 / 5}$ and $d=0.37 \xi^{6 / 5} L_{p}^{-1 / 5}$ [Hinner et al. (1998)]. Only close to the entanglement concentration, which is somewhat higher than the naive overlap concentration, do the dangling ends become important.

\section{B. High frequency behavior}

At frequencies above the "rubber plateau" (i.e., roughly above $1 \mathrm{~Hz}$ for typical F-actin solutions of a concentration of about $1 \mathrm{mg} / \mathrm{ml}$ ) there is a pronounced increase in the storage modulus. Several previous experimental publications on $\mathrm{F}$-actin solutions at $C \approx 1 \mathrm{mg} / \mathrm{ml}$ report a storage modulus which rises as $\omega^{3 / 4}$ at high frequencies. However, not many of these reports clarify the frequency range over which this scaling is observed. Forced magnetic bead microrheology has reported $G^{\prime}(\omega) \sim \omega^{3 / 4}$ between $10^{1} \mathrm{~s}^{-1}$ $\leqslant \omega \leqslant 10^{2} \mathrm{~s}^{-1}$ [Amblard et al. (1996)]. Measurements using one-dimensional interferometric microrheology resulted in a report of $G^{\prime}(\omega) \sim \omega^{3 / 4}$ also between $10^{1} \mathrm{~s}^{-1}$ $\leqslant \omega \leqslant 10^{2} \mathrm{~s}^{-1}$ [Gittes et al. (1997)]. Other DWS measurements of F-actin solutions [Xu et al. (1998a, 1998b); Palmer et al. (1999)] have been interpreted in terms of a very abrupt transition in $G^{\prime}(\omega)$ from a constant low frequency plateau to a $\omega^{3 / 4}$ dependence for $10^{2} \mathrm{~s}^{-1} \leqslant \omega \leqslant 10^{3} \mathrm{~s}^{-1}$. The analysis of the DWS data used to determine the moduli in Palmer et al. (1999) appears to be only a patch of low and high frequency asymptotic limits [the overlapping of the asymptotic limits in their $G^{\prime}(\omega)$ is evident in Fig. 7 of Palmer et al. (1999) between $10^{1} \mathrm{~s}^{-1} \leqslant \omega \leqslant 10^{2} \mathrm{~s}^{-1}$ ] and their $G^{\prime}(\omega)$ in the crossover 
region appear to be kinked rather than smooth as a result of the patching. Finally, we have reported DWS measurements that include the effects of an $\omega^{1 / 3}$ term that is required to fit the more gradual observed crossover region between the low frequency plateau and the high frequency scaling $G^{\prime}(\omega) \sim \omega^{3 / 4}$ [Gisler and Weitz (1999)]; this high frequency scaling begins to completely dominate the other two terms for $\omega \geqslant 10^{4} \mathrm{~s}^{-1}$. The more detailed regularization analysis presented in this work is in agreement with this previous conclusion based on analytic continuation using a fitting form, and $G^{\prime}(\omega) \sim \omega^{3 / 4}$ is clearly not the observed behavior for $\omega<10^{4} \mathrm{~s}^{-1}$ for $C \approx 1 \mathrm{mg} / \mathrm{ml}$.

\section{Crossover behavior}

Although we believe that the gradual crossover can be explained without appealing to the nonideality of the sample, as we discuss in the following paragraphs, we cannot rule out the possibility that the differences in the reported breadths of the crossover could be due to different sample preparation methods that lead to different polydispersities in the filament length distribution and inhomogeneities in the filament concentration. However, the gradual crossover we report is not due to our choice of analytical method. In principle, all transformation methods, whether the regularization method presented here, the analytic continuation method in Gisler and Weitz (1999), or the dispersion integral method in Gittes et al. (1997), should yield the same broad crossover if they accurately reflect the measured $J(t)$ shown in Fig. 1, since they are all based on the KramersKronig relations. Any of these methods may break down if there is significant noise in the measured $\left\langle\Delta r^{2}(t)\right\rangle$ or if the frequencies of interest correspond to the temporal extremes of the available data. For a general overview of numerical transformation methods between $J(t)$ and $G^{*}(\omega)$, the interested reader can consult Ferry (1980).

A comparison of the $G^{\prime}(\omega)$ and $G^{\prime \prime}(\omega)$ we have measured with recent theoretical predictions for the high frequency viscoelastic response [Gittes and MacKintosh (1998); Morse (1998a, 1998b, 1998c)] suggests that although there is agreement of theory and experiments concerning the limiting behavior, a closer theoretical look is needed to explain the crossover region between the low frequency elastic plateau and the high frequency $\omega^{3 / 4}$ power law. Potential problems of these theoretical models could arise from the ad hoc assumptions regarding the nature of the force transmission and/or the static tube concepts, which might lead to unrealistic predictions in the crossover region.

A theory is needed that links the static entanglement models for the plateau modulus with the single polymer dynamics at high frequencies. In general, there may be a subtle interplay between collective modes on long length scales and single polymer modes on short length scales. Despite this potentially complicated interplay, the retardation spectrum $L(\tau)$ in Fig. 2 reveals a simple underlying structure. If we tentatively attribute a physical significance to the basis functions in Eq. (3) as overdamped "phonons" of the polymer network, $L(\tau)$ would be the analogy of the density of states in solid state physics. From this point of view, one could say that the eigenmode spectrum of the system is simple. It behaves like a scaling function with a kink at a characteristic time $\tau^{*} \sim 10^{-1} \mathrm{~s}$. The limits of this scaling function for long and short times are related to the plateau regime and the high frequency modulus, respectively. As we have pointed out, there seems to be reasonable agreement between theory and experiments about these limits but not about the form of the crossover. As revealed by Fig. 2, the experimentally observed broad crossover in the moduli corresponds to the kink at $\tau^{*}$ in $L(\tau)$. The moduli obtained by working backwards from an idealized retardation spectrum that obeys $L\left(\tau<\tau^{*}\right) \sim \tau^{3 / 4}$ and $L\left(\tau>\tau^{*}\right) \sim \tau^{-1 / 2}$ (see Fig. 2) are shown together with those obtained directly from the data in Fig. 4. The overall agreement is quite good and can be improved by averaging over many independent experiments [see the curves in Gisler and 
Weitz (1999)]. Nevertheless, there are still some differences between the moduli based on the measurements and the moduli obtained from the idealized retardation spectrum. At low frequencies, these differences may be due to the breakup of the polymer cages around the beads and eventually to the viscous long-time relaxation of the solution. At high frequencies, these difference may be due to an imperfect coupling of the beads to the polymer network; since the penetration depth of a dynamic excitation into the pure solvent is only about $1 \mu \mathrm{m}$ at $10^{6} \mathrm{~s}^{-1}$, we are not very far from the limit where the thermal motion of the beads is dominated by the solvent. All of these features imply that, beneath the complicated problem of the viscoelasticity of semiflexible polymer solutions, there lies a simple structure of the retardation spectrum, which is not explained by the existing theoretical models.

Finally, in future experiments, it would be intriguing to study how shear changes the filament structure and low frequency relaxation immediately after quenching the G-actin solution with the divalent salt solution. It would also be desirable to measure the interactions between the actin filaments and the probe spheres; if the spheres are strongly attracted to the actin filaments, so that they have "hairs" of bound filaments which extend many particle diameters away, then the continuum approximation may not be far off, although the effective radius of the sphere may be uncertain. This would be consistent with the smaller $G_{p}^{\prime}$ given by the optical measurements; spheres with protruding hairs of actin filaments would have a larger effective radius and thus a correspondingly smaller $G_{p}^{\prime}$ as predicted by Eq. (1) and in accord with the mechanical measurements. To test this hypothesis, one could control the surface chemistry (e.g., positively versus negatively charged) of the probe spheres and note possible differences in the mean square displacement.

\section{CONCLUSION}

These optical tracer measurements of the storage and loss moduli of an F-actin solution show the general rheological features that characterize semiflexible polymer solutions in the semidilute limit over a very wide frequency range. There is a gradual crossover in the storage modulus from a dominant low frequency plateau elasticity to a high frequency scaling behavior consistent with $G^{\prime}(\omega) \sim \omega^{3 / 4}$. This gradual crossover translates, however, to a kink between two scaling branches of the retardation spectrum $L(\tau)$, which reveals a surprisingly simple structure of the mode spectrum of the system. Explaining its origin remains a challenging problem for further theoretical investigations.

The plateau elasticity of reconstituted pure F-actin solutions at $C$ near typical cellular concentrations lies at least several orders of magnitude below a typical elasticity $G_{p}^{\prime}$ $\approx 10^{4}$ dynes $/ \mathrm{cm}^{2}$ of a whole white blood cell [Evans et al. (1993)]. This is not surprising because F-actin in the cytoplasm can be bundled and crosslinked by proteins such as fascin and $\alpha$-actin [Pollard and Cooper (1986)]. Microtubulues and intermediate filaments may lend additional structural support. Because the elastic modulus of a purified F-actin solution at cellular concentrations is much lower than that of a whole cell, other proteins, not just F-actin, play an important role in setting the viscoelasticity of the cytoplasm [Xu et al. (1999)]. In the future, microrheological particle tracking methods applied to naturally occurring organelles or artificially introduced particles within the cytoplasm may help unravel how structural and regulatory proteins affect the viscoelasticity of the cytoplasm and influence intracellular transport. 


\section{ACKNOWLEDGMENTS}

The authors thank D. Morse, F. Gittes, and F. MacKintosh for stimulating discussions.

\section{References}

Amblard, F., A. C. Maggs, B. Yurke, A. N. Pargellis, and S. Leibler, "Subdiffusion and anomalous local viscoelasticity in actin networks," Phys. Rev. Lett. 77, 4470-4473 (1996).

Bird, R. B., R. C. Armstrong, and O. Hassager, Dynamics of Polymeric Liquids (Wiley, New York, 1977).

Evans, E., A. Leung, and D. Zhelev, "Synchrony of cell spreading and contraction force as phagocytes engulf large pathogens," J. Cell Biol. 122, 1295-1300 (1993).

Ferry, J. D., Viscoelastic Properties of Polymers (Wiley, New York, 1980).

Frey, E., K. Kroy, and J. Wilhelm, "Physics of solutions and networks of semiflexible macromolecules and the control of cell function," in The Wiley Polymer Networks Group Review Series, edited by B. T. Stokke (Wiley, New York, 1999), Vol. 2.

Gisler, T. and D. A. Weitz, "Scaling of the microrheology of semidilute F-actin solutions," Phys. Rev. Lett. 82, 1606-1609 (1999).

Gittes, F. and F. MacKintosh, “Dynamic shear modulus of a semiflexible polymer network," Phys. Rev. E 58, R1241-R1244 (1998).

Gittes, F., B. Schnurr, P. D. Olmsted, F. C. MacKintosh, and C. F. Schmidt, "Microscopic viscoelasticity: Shear moduli of soft materials determined from thermal fluctuations," Phys. Rev. Lett. 79, 3286-3289 (1997).

Helfrich, W. and W. Harbich, "Adhesion and cohesion of tubular vesicles," Chem. Scr. 25, 32-36 (1985).

Hinner, B., M. Tempel, E. Sackmann, K. Kroy, and E. Frey, "Entanglement, elasticity, and viscous relaxation of actin solutions," Phys. Rev. Lett. 81, 2614-2617 (1998).

Isambert, H. and A. C. Maggs, "Dynamics and rheology of actin solutions," Macromolecules 29, 1036-1040 (1996).

Janmey, P. A., S. Hvidt, J. Käs, D. Lerche, A. C. Maggs, E. Sackmann, M. Schliwa, and T. P. Stossel, "The mechanical properties of actin gels,' J. Biol. Chem. 269, 32503-32513 (1994).

MacKintosh, F. C., J. Käs, and P. A. Janmey, "Elasticity of semiflexible biopolymer networks," Phys. Rev. Lett. 75, 4425-4428 (1995).

Mason, T. G. and D. A. Weitz, "Optical measurements of the linear viscoelastic moduli of complex fluids," Phys. Rev. Lett. 74, 1250-1253 (1995).

Mason, T. G., H. Gang, and D. A. Weitz, "Optical measurements of the linear viscoelastic moduli of complex fluids," J. Opt. Soc. Am. A 14, 139-149 (1997).

Morse, D. C., "Viscoelasticity of concentrated isotropic solutions of semiflexible polymers: (1) Model and stress tensor," Macromolecules 31, 7030-7043 (1998a).

Morse, D. C., "Viscoelasticity of concentrated isotropic solutions of semiflexible polymers: (2) Linear response," Macromolecules 31, 7044-7067 (1998b).

Morse, D. C., "Viscoelasticity of tightly entangled solutions of semiflexible polymers," Phys. Rev. E 58, R1237-R1240 (1998c).

Odijk, T., "Theory of lyotropic liquid crystals," Macromolecules 19, 2313-2329 (1986).

Onsager, L., "The effects of shape on the interaction of colloidal particles," Ann. (N.Y.) Acad. Sci. 51, $627-659$ (1949).

Palmer, A., J. Xu, S. C. Kuo, and D. Wirtz, "Diffusing wave spectroscopy microrheology of actin filament networks," Biophys. J. 76, 1063-1071 (1999).

Pollard, T. D. and J. A. Cooper, "Actin and actin-binding proteins. A critical evaluation of mechanisms and functions," Annu. Rev. Biochem. 55, 987-1035 (1986).

Press, W. H., S. A. Teukolsky, W. T. Vetterling, and B. P. Flannery, Numerical Recipies in C (Cambridge University Press, Cambridge, 1992).

Semenov, A. N., "Dynamics of concentrated solutions of rigid-chain polymers. Part 1. Brownian motion of persistent macromolecules in isotropic solution,' J. Chem. Soc., Faraday Trans. 1 86, 317-329 (1986).

Spudich, J. and S. Watt, "The regulation of rabbit skeletal muscle contraction," J. Biol. Chem. 246, 4866-4871 (1971).

Weitz, D. A. and D. J. Pine, "Diffusing wave spectroscopy," in Dynamic Light Scattering, edited by W. Brown (Oxford University Press, Oxford, 1992).

Xu, J., The Mechanical and Physical Properties of Actin Filament Networks (Johns Hopkins University Press, Baltimore, MD, 1997).

Xu, J., J. F. Casella, and T. D. Pollard, "Effect of capping protein, CapZ, on the length of actin filaments and mechanical properties of actin filament networks," Cell Motil. Cytoskeleton 42, 73-81 (1999). 
Xu, J., W. H. Schwarz, J. A. Käs, T. P. Stossel, P. A. Janmey, and T. D. Pollard, "Mechanical properties of actin filament networks depend on preparation, polymerization conditions, and storage of actin monomers," Biophys. J. 74, 2731-2740 (1998a).

Xu, J., V. Viasnoff, and D. Wirtz, "Compliance of actin filament networks measured by particle tracking microrheology and diffusing wave spectroscopy," Rheol. Acta 37, 387-398 (1998b).

Ziemann, F., J. Rädler, and E. Sackmann, "Local measurements of viscoelastic moduli of entangled actin networks using an oscillating bead micro-rheometer," Biophys. J. 66, 2210-2216 (1994). 